\title{
Caracterización de trauma arterial periférico en un hospital de tercer nivel
}

\author{
Characterization of peripheral arterial trauma at a third level of care hospital
}

\author{
Héctor Jiménez 1,2, Edwin Romero3, Rolando Medina4, Wilmer Botache ${ }^{4,5}$, Juan Sanjuán 1,2, \\ Carlos Martínez ${ }^{6}$, Adriana Morales ${ }^{6}$, Alejandra Duran ${ }^{6}$, Francisco Montoya ${ }^{6}$, Jhon Salamanca ${ }^{7}$ \\ Médico, residente de Cirugía general. Universidad Surcolombiana, Neiva, Colombia. \\ 2 Especialista en Epidemiología \\ 3 Médico, cirujano vascular periférico. Departamento de Cirugía general, Universidad Surcolombiana, Neiva, Colombia. \\ 4 Médico cirujano, cirujano de trauma y cuidados intensivos. Departamento de Cirugía general, Universidad Surcolombiana, Neiva, \\ Colombia. \\ 5 Coordinador grupo de investigación CYTRA. \\ 6 Médico general, Universidad Surcolombiana. Neiva, Colombia \\ 7 Médico, residente de Medicina Interna. Universidad Surcolombiana, Neiva, Colombia.
}

\section{Resumen}

Introducción. El trauma vascular es un evento de baja frecuencia, con alta carga de morbilidad y mortalidad, que afecta mayormente a hombres jóvenes y en muchas ocasiones requiere manejo quirúrgico. Se asocia con múltiples complicaciones, que van desde la reintervención quirúrgica, hasta la amputación de la extremidad, las cuales, a su vez, se ven influenciadas por variables relacionadas tanto con el trauma como con la atención hospitalaria. Objetivo. Caracterizar los pacientes mayores de I3 años con traumatismos arteriales periféricos en el Hospital Universitario Hernando Moncaleano Perdomo, de Neiva, Huila, Colombia, en el periodo enero de 2014 a diciembre de 2016.

Materiales y métodos. Estudio observacional, retrospectivo, de tipo descriptivo, con población a conveniencia de pacientes mayores de 13 años que sufrieron traumatismos arteriales periféricos y fueron llevados a procedimiento quirúrgico en el Hospital Universitario Hernando Moncaleano Perdomo.

Resultados. Se encontraron 54 pacientes con trauma arterial periférico, cifra que representa el I,5\% de los ingresos por trauma en la Institución durante el período de estudio. El género más representativo fue el masculino $(n=49)$, las heridas por arma cortopunzante el principal mecanismo de trauma $(n=22)$ y las extremidades el área comúnmente afectada $(\mathrm{n}=47)$. Se halló un puntaje de gravedad de la lesión promedio al ingreso de 26 puntos, y un tiempo de isquemia de extremidad mayor a seis horas al momento del ingreso en el 31,5\% de los casos ( $\mathrm{n}=\mathrm{I} 7)$. Conclusiones. Las intervenciones quirúrgicas fueron hechas en mayor medida por cirugía de trauma, seguido de cirugía vascular. El principal manejo fue la anastomosis término-terminal y el injerto de safena invertida. Las complicaciones fueron en mayor medida la trombosis arterial $(n=I 2)$, seguida de la amputación de extremidad ( $n=7)$. Palabras clave: lesiones del sistema vascular; heridas penetrantes; traumatismo múltiple; extremidad inferior; extremidad superior; procedimientos quirúrgicos vasculares.

Fecha recibido: 14/12/2017 - Fecha aceptación: 22/05/2018

Correspondencia: Carlos Mauricio Martínez. Calle 80B \# 2C-13 Barrio Santa Rosa, Neiva, Huila, Colombia. Teléfono: 320-4016821. Correo electrónico: carlitos220792@gmail.com

Citar como: Jiménez HC, Romero E, Medina R, Botache W, Sanjuán J, Martínez C, Morales A, Duran A, Montoya F, Salamanca J. Caracterización de trauma arterial periférico en un hospital de tercer nivel. Rev Colomb Cir. 2018;33:272-79. https://doi.org/10.30944/20117582.72 


\begin{abstract}
Introduction. Vascular trauma is a low frequency event, with a high burden of morbidity and mortality that mostly affects young men. It often requires surgical management, which is associated with multiple complications ranging from reoperation to limb amputation; that in turn, are influenced by variables associated with both trauma and hospital care.

Objective. To characterize the patients over 13 years of age with peripheral arterial trauma admitted to the Hernando Moncaleano Perdomo University Hospital, Neiva, Colombia, from January 2014 to December 2016.

Methods. Retrospective observational study of descriptive type with population of patients older than 13 years with peripheral arterial trauma who had a surgical procedure at the Hernando Moncaleano Perdomo University Hospital.

Results. This study showed that the 54 patients with peripheral arterial trauma represented $1.5 \%$ of the total trauma admissions at the Institution during the study period. The most representative gender was male $(n=49)$, short stab wounds were the main mechanism of trauma $(n=22)$, and the extremities were the most commonly affected area $(n=47)$. An average ISS (Injury Severity Score) and a limb ischemia time of more than 6 hours in $31.48 \%(n=17)$ of the patients were recorded at entry.
\end{abstract}

Conclusions. Surgical interventions were undertaken by trauma surgery followed by vascular surgery. The main postoperative complications were arterial thrombosis $(n=12)$ followed by limb amputation $(n=7)$.

Key words: vascular system injuries; wounds, penetrating; multiple trauma; lower extremity; upper extremity; vascular surgical procedures.

\section{Introducción}

El trauma vascular es un evento de baja frecuencia, que se relaciona con alta carga de morbilidad y mortalidad ${ }^{\mathrm{I}}$ La incidencia reportada en diferentes experiencias internacionales oscila entre el o,3 al $3 \%$; en el contexto local (Colombia) es del 0,3 al 2I,2 \% ${ }^{\mathrm{I} \cdot 4}$. En general, las lesiones vasculares se describen en hombres jóvenes con un mecanismo predominantemente penetrante, $y$ variación en cuanto a severidad, presentación y compromiso de otros sistemas de acuerdo con las características y el sitio del trauma ${ }^{5}$. En mayor medida se comprometen las extremidades inferiores (3I - $36 \%$ ), seguidas de las extremidades superiores ( $\mathrm{I} 8,4-3 \mathrm{I} \%)$, los vasos abdominales y pélvicos (I5,6 - 2I \%), el cuello (IO - 24,8\%) y el tórax (IO - I3,8\% $)^{3,5,6}$. Se han descrito la severidad y el mecanismo de las lesiones en relación con la necesidad de manejo quirúrgico, que varía entre 2 a $60 \%$ de todas las lesiones vasculares ${ }^{1,2}$. Entre los procedimientos requeridos en la reparación de estas lesiones están las ligaduras vasculares, los reparos primarios y el baipás o la embolización selectiva; tales procedimientos generan complicaciones postquirúrgicas como trombosis o estenosis del reparo $(3 \%)$, infección del sitio operatorio con necesidad de reintervención (I,I \%), reintervención por alguna causa relacionada con el procedimiento inicial $(7,7 \%)$ y amputaciones $(3,3-29 \%)^{7,8}$. La mortalidad relacionada con estas lesiones es de aproximadamente un $2-22,9 \%$ 5,6,9, valores que cambian de acuerdo con variables como la energía del mecanismo de la lesión, el tiempo de evolución a la atención, el sitio de la lesión, las lesiones asociadas, el tipo de reparo y las características operativas de la cirugía inicial, entre otras ${ }^{2, \mathrm{IO}}$; se han relacionado además como influyentes en la aparición de complicaciones tras el reparo de las lesiones vasculares.

En relación a esto y a la variabilidad en la presentación del trauma de acuerdo con las regiones, los mecanismos y las características de las poblaciones, se plantea la necesidad de describir las características de los pacientes que fueron atendidos por trauma vascular arterial quirúrgico en el Hospital Universitario Hernando Moncaleano Perdomo de la ciudad de Neiva en el período 2014 a 2016.

\section{Materiales y métodos}

Estudio observacional, retrospectivo, de tipo descriptivo, que incluyó población a conveniencia, 
obtenida mediante la búsqueda activa de historias clínicas y revisión de informes quirúrgicos de pacientes con trauma arterial periférico llevados a procedimiento quirúrgico por parte de los servicios de Cirugía general, Trauma y Vascular del Hospital Universitario Hernando Moncaleano Perdomo, en el período comprendido entre 20I4 a 2016. Se obtuvo una población de 54 pacientes, excluyendo todos aquellos con historias clínicas incompletas y pacientes menores de I3 años.

\section{Características de la atención}

Todos recibieron atención inicial con base en las guías del Soporte Vital Avanzado en Trauma (del inglés Advanced Trauma Life Support o ATLS $^{\circledR}$ ) dadas las políticas institucionales ${ }^{\mathrm{I}}$. Como parte del protocolo institucional establecido para los pacientes con lesiones traumáticas, se determina que una vez son atendidos en el servicio de urgencias, se solicita valoración oportuna por el servicio de cirugía general donde se realiza la segunda evaluación dirigida. Las intervenciones vasculares pueden ser hechas por cirujanos generales, cirujanos de trauma o cirujanos vasculares de acuerdo con la pertinencia y disponibilidad de los servicios. La decisión del tipo de reparación quirúrgica, se basa en el mecanismo, la presentación y la clínica del paciente.

Las variables estudiadas en esta población incluyeron datos sociodemográficos, comorbilidades, causa de la lesión, área anatómica afectada, parámetros fisiológicos al ingreso, signos clínicos de trauma vascular, puntaje de gravedad de la lesión (del inglés Injury Severity Score o ISS), tiempo de isquemia de la extremidad, hallazgos intraoperatorios (arteria y tercio arterial comprometido, tipo de lesión, lesiones asociadas), especialista encargado del procedimiento quirúrgico, tipo de reparación vascular, complicaciones posquirúrgicas y necesidad de manejo en cuidados intensivos. Como variable de respuesta se estudiaron los resultados postquirúrgicos a un mes.

Los datos se incorporaron en una matriz creada en Microsoft Excel y se procesaron en el programa estadístico SPSS (versión de prueba) versión 23, con el cual se generaron valores descriptivos (frecuencias y proporciones) para las variables cualitativas, medidas de tendencia central y medidas de dispersión para las variables cuantitativas. Antes de realizar el contraste de hipótesis se comprobó si las variables cuantitativas tenían una distribución normal mediante la prueba de Kolmogorov-Smirnov.

\section{Resultados}

\section{Sociodemográficos}

Del total de pacientes atendidos en el Hospital Universitario Hernando Moncaleano Perdomo entre los años 2014 y 20I6, 3.650 casos (26\%), ingresaron con algún diagnóstico relacionado a trauma, de los cuales 54 (I,5\%) tuvieron algún tipo de trauma arterial periférico que requirió manejo quirúrgico; esta constituyó la población de pacientes incluidos en este estudio.

Del total de pacientes incluidos, 49 eran hombres (90,7\%), tenían edad promedio de 28 años (media de 26,5 años) y correspondían a los estratos socioeconómicos I y 2 , principalmente. Las comorbilidades más comunes asociadas a esta población fueron la farmacodependencia, en 6 pacientes (II,I \%), seguida por la hipertensión arterial, en 2 pacientes (3,7 \%).

\section{Características clínicas de la lesión arterial}

Dentro de las principales causas de lesión arterial periférica se encontraron las heridas por arma cortopunzante, en 22 pacientes (40,7\%), las heridas por arma cortocontundente, en I2 pacientes $(22,2 \%)$, seguido por heridas por proyectil de arma de fuego, en Io pacientes (I8,5\%) y por último los accidentes de tránsito, específicamente relacionados con motocicletas, en 8 pacientes.

La principal región anatómica afectada por los diferentes traumas fueron las extremidades, en un $87 \%$ de los casos, la cual se encontró asociada a traumas en otras regiones corporales como el tórax, en 13 pacientes (24,6\%), la cabeza, en 7 pacientes (I3,I \%) y el abdomen, en 6 casos (II,I \%). Respecto a las variables fisiológicas de estos pacientes, a su ingreso tuvieron promedio de presión arterial media de 77,3 $\mathrm{mm} \mathrm{Hg}(64-92 \mathrm{~mm}$ $\mathrm{Hg}$ ), frecuencia respiratoria promedio de 23 (IO - 35) respiraciones por minuto y una calificación en la escala de coma de Glasgow de I3 (I4,3-I5). 
El diagnóstico clínico de los pacientes con trauma arterial periférico, se enfocó en la descripción al examen físico de signos duros y blandos de trauma vascular; dentro de los signos duros, los más frecuentes fueron el sagrado pulsátil, seguido por la ausencia de pulsos y el hematoma expansivo; en cuanto a los signos blandos la mayoría de los pacientes presentó herida en trayecto vascular (figura I).

\section{Manejo de las lesiones arteriales periféricas}

Un total de 40 pacientes $(74, \mathrm{I} \%)$ recibieron intervención quirúrgica a cargo del servicio de Cirugía general, 9 y 5 pacientes fueron tratados por los servicios de Cirugía vascular y Cirugía de trauma, respectivamente. Esta población tuvo en promedio un puntaje de gravedad de la lesión de 26 puntos ( 13 - 30); además, I7 pacientes $(3 \mathrm{I}, 4 \%)$

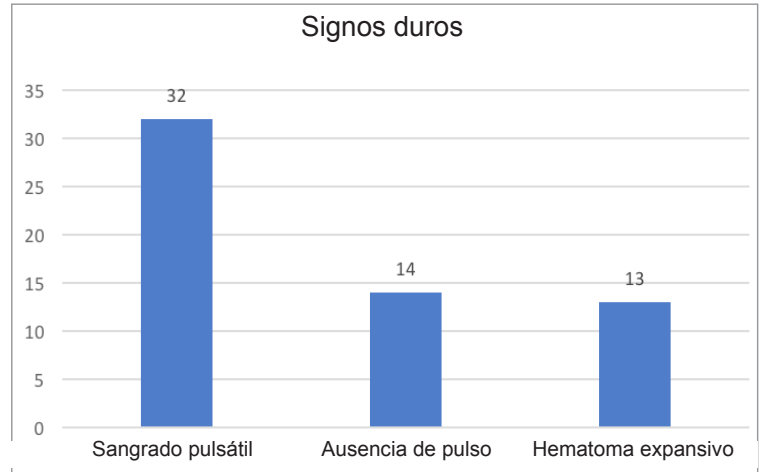

tuvieron un tiempo de hipoperfusión de la extremidad (tiempo de isquemia), mayor a 6 horas (promedio $=9,05$ horas).

Dentro de los hallazgos intraoperatorios, a 3I pacientes se les realizó una exploración vascular exclusiva $(57,4 \%)$ y 23 requirieron otros procedimientos (42,6\%), 8 debido a lesiones como fracturas óseas $(34,7 \%$ ) y I5 debido a avulsión de tejidos y lesiones nerviosas $(65,3 \%)$. Los principales trayectos arteriales comprometidos se enuncian en la figura 2. El sitio de lesión y reparación arterial en los pacientes con trauma arterial periférico fue el tercio proximal, en 20 casos (37\%), el medio en $19(35,2 \%)$ y el distal en 15 ( $27,8 \%)$. Así mismo, se pudo identificar que el tipo de lesión arterial más frecuente fue la transección, en 28 pacientes (5I,9\%), seguida de la sección parcial en el I8,5\% (figura 3).

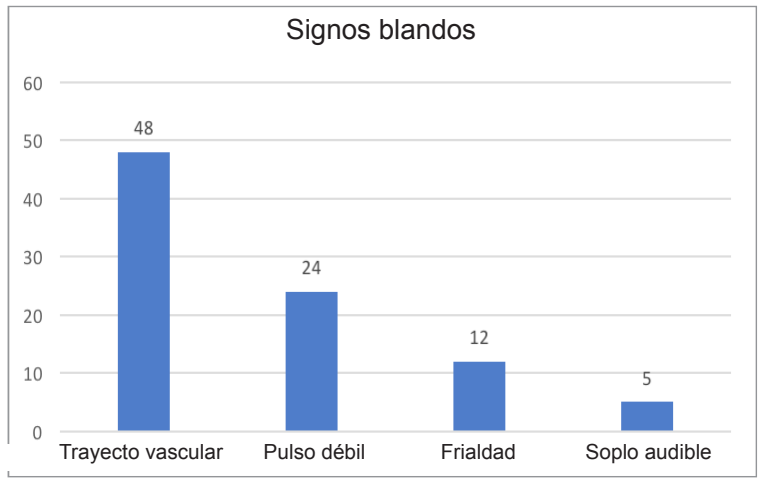

Figura 1. Signos clínicos de trauma vascular comunes en pacientes con trauma arterial periférico admitidos en el Hospital Universitario Hernando Moncaleano Perdomo, 2014 - 2016.

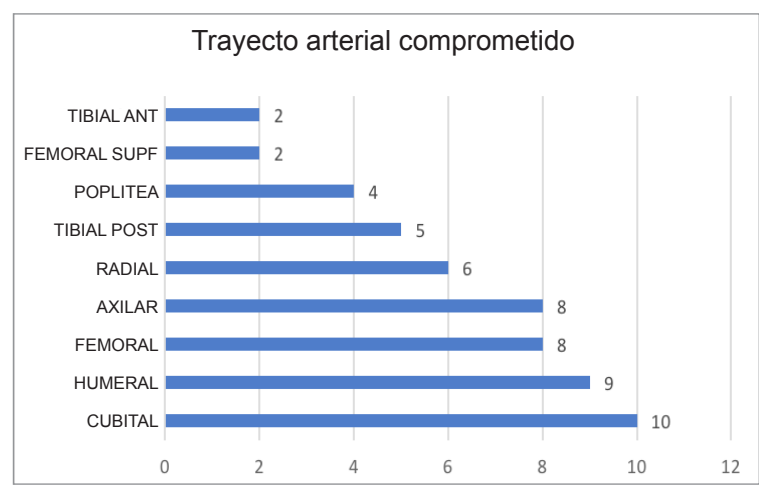

Figura 2. Trayectos arteriales comprometidos en pacientes con trauma vascular periférico del Hospital Universitario Hernando Moncaleano Perdomo 2014 - 2016.

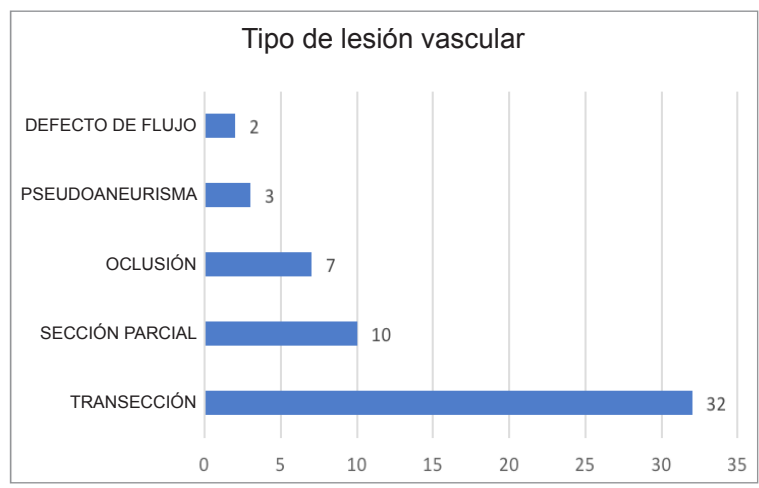

Figura 3. Tipos de lesiones arteriales en los pacientes con trauma arterial periférico del Hospital Universitario Hernando Moncaleano Perdomo 2014 - 2016. 
Se realizó anastomosis término - terminal a 24 pacientes $(44,4 \%)$, reconstrucción con injerto invertido de safena a 19 (35,I \%) y ligadura vascular a II (20,3\%) (figura 4).

Posterior al tratamiento quirúrgico, el tiempo de estancia hospitalaria de esta población fue en promedio 9,7 días; de 54 pacientes, I4 $(27,4 \%)$ requirieron manejo complementario e integral en unidad de cuidados intensivos, con una estancia promedio de 6,42 días; 19 pacientes necesitaron soporte inotrópico por 3,92 días en promedio. En cuanto a complicaciones posoperatorias la más frecuente fue la trombosis arterial, en I2 pacientes $(22,2 \%)$, seguido por amputación, en 7 pacientes (I3\%), trombosis del injerto venoso, en 6 pacientes (II,I \%), infecciones del sitio operatorio y muerte, en 3 pacientes (5,6\%). Finalmente, de todos los pacientes, I4 (25,9\%), necesitaron una reintervención quirúrgica, en un promedio de 8,42 horas posoperatorias.

\section{Discusión}

El trauma, tanto en el mundo como en Colombia, específicamente en el departamento del Huila, es una de las consultas más frecuentes en los servicios de urgencias ${ }^{\mathrm{I}}$. Aunque el traumatismo cerrado es una causa común de la presentación de trauma agudo en la población civil en todo el mundo, la creciente violencia urbana en nuestra región ha producido un aumento del trauma penetrante producido por disparos con arma de fuego y heridas por arma blanca ${ }^{5, \mathrm{IO}, \mathrm{II}}$. Es así como el Hospital Universitario Hernando Moncaleano Perdomo, de la ciudad de Neiva, se ha convertido en un centro de referencia para la atención y el manejo respectivo de los pacientes víctimas de trauma, y dado que cuenta con el servicio de Cirugía vascular periférica, es el punto de manejo específico del trauma vascular periférico en el Sur de Colombia.

Los datos de la prevalencia del trauma vascular periférico en las series mundiales y diferentes estudios, es baja; oscila entre el o,65 al I,4 \% del total de las consultas ${ }^{\mathrm{I}, \mathrm{II}, 12}$, con algunas excepciones como los traumas vasculares en zonas de combate, cuya prevalencia está entre el $\mathrm{I} 7 \mathrm{al} 24 \%{ }^{8}$. El presente estudio mostró una prevalencia de I,5\% (54 pacientes en 3 años de seguimiento), situación que pone en evidencia la alta tasa de trauma vascular (arterial periférico) que acaece en el departamento del Huila, y la importancia de poseer experiencia y conocimientos adecuados y actualizados sobre el tratamiento de este tipo de trauma.

De acuerdo con los resultados de este estudio, la población más afectada por trauma arterial periférico son los pacientes jóvenes, con edad promedio de 26 años, socialmente productiva, principalmente del género masculino, a causa de lesiones arteriales secundarias, especialmente de heridas por arma cortopunzante y cortocontundente, datos similares a los reportados por

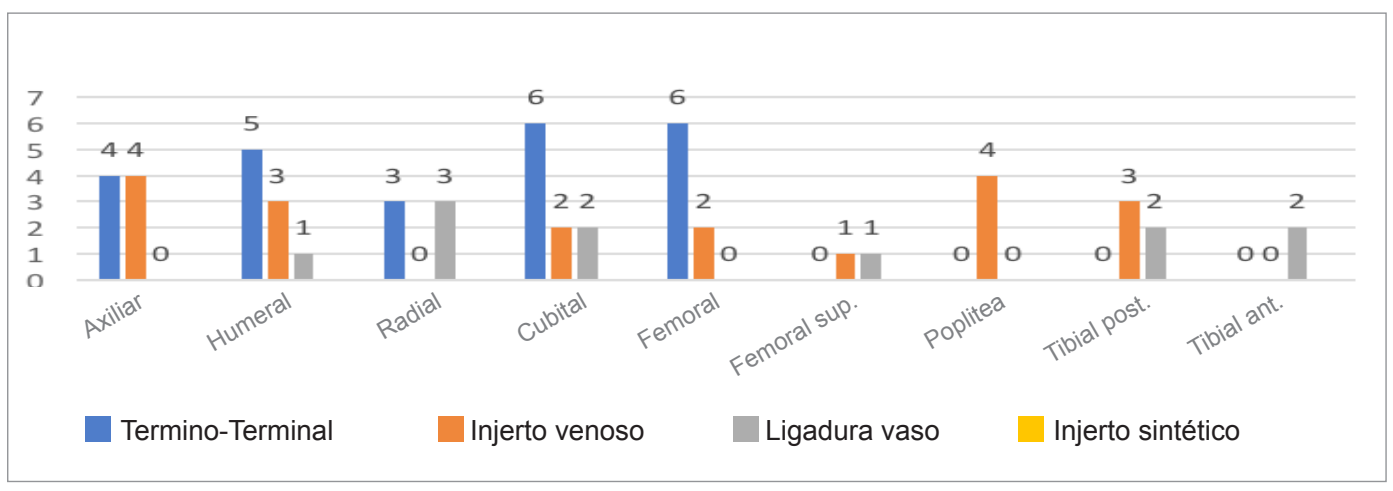

Figura 4. Tipo de reparación según arteria comprometida en pacientes con trauma arterial periférico del Hospital Universitario Hernando Moncaleano Perdomo 2014 - 2016 
diferentes autores nacionales e internacionales $3,5,7,1,1,1,1,13-15$.

En los hallazgos clínicos de los pacientes objeto de estudio, el principal signo duro de trauma vascular fue el sangrado pulsátil (59,3\%), seguido de ausencia de pulso $(25,9 \%)$ y hematoma expansivo (24\%); respecto a los signos blandos, el principal fue la herida en trayecto vascular; una cohorte de 92 pacientes muestra que la presencia de signos duros fue de un $77 \%$, en cuyo caso la ausencia de pulso fue el principal signo (40 \% de los pacientes), seguido de sangrado pulsátil $(26,8 \%)$ y hematoma expansivo $(2,3 \%)^{\mathrm{IO}, 16}$. Otros estudios muestran una prevalencia de signos duros del $29,3 \%$, dato que es inferior a lo observado en nuestro estudio?.

En lo concerniente a los resultados sobre hallazgos intraoperatorios, se evidencian lesiones con compromiso de extremidades, mayormente de miembros superiores (arteria cubital y radial), seguida por lesiones en miembros inferiores (arteria femoral común), datos que van de la mano con resultados de estudios locales e internacionales ${ }^{3-5,10,14}$. Respecto a otra literatura internacional, algunos estudios muestran que la principal región afectada son las extremidades, pero en porcentajes menores a los observados en nuestro estudio (59 vs. $87 \%$ respectivamente) ${ }^{\mathrm{I}}$, mientras que otros muestran que las principales regiones afectadas son la cabeza y el cuello; la arteria carótida interna es la más comprometida ${ }^{7}$.

Las principales lesiones arteriales encontradas fueron la transección, seguida por la oclusión del vaso (trombos), datos concordantes con la literatura ${ }^{4,7}$. En la mayoría de los casos las lesiones anteriores requirieron reparación primaria (anastomosis término-terminal), en tanto que el uso de injerto invertido de safena, datos similares a los reportados en la literatura ${ }^{7,10,1,1,17}$, dependió de la magnitud del trauma, la pérdida de longitud arterial y el estado de los cabos distal y proximal del segmento afectado.

En esta institución de tercer nivel del departamento del Huila, el trauma vascular fue atendido principalmente por el servicio de Cirugía general, a excepción de algunos casos que fueron manejados por servicios como Cirugía de trauma y Cirugía vascular periférica ( 5 y 9 pacientes, respectivamente), contrario a algunos trabajos de la literatura mundial en los que se menciona un mayor porcentaje de atención de este tipo de trauma por parte del servicio de Cirugía vascular ${ }^{1,4}$, fenómeno que se explica por las mejores condiciones de los sistemas de salud en el ámbito internacional y la disponibilidad de este servicio las 24 horas en algunas instituciones. De acuerdo con la magnitud del trauma y las lesiones asociadas, el $27,4 \%$ de esta población requirió un manejo en la unidad de cuidados intensivos.

De los traumas arteriales atendidos en este Hospital, debido al lugar de procedencia, en algunos de casos de áreas rurales y centros de atención de primer nivel distantes, el 3I,5\% tuvo tiempos de isquemia crítica de las extremidades afectadas, mayor a 6 horas (promedio 9 horas), situación que aunque no se concluyó estadísticamente en este estudio, pudo influir directamente en el pronóstico y los resultados posquirúrgicos de estos pacientes, apoyado en las conclusiones de algunos estudios internacionales en los que se aclara la importancia de la revascularización de las lesiones arteriales en las primeras 6 horas posteriores al trauma, logrando así mejores resultados posquirúrgicos y menores tasas de pérdida de extremidades ${ }^{\text {II }}$. Igualmente, se logró identificar a la trombosis arterial y a la amputación como las principales complicaciones posoperatorias de esta población; cabe resaltar la alta tasa de amputación, la cual duplica significativamente las obtenidas en el ámbito internacional (5\%), según datos reflejados en diferentes estudios ${ }^{12,16}$.

Todo lo anterior permite evidenciar la importancia que tiene el trauma arterial periférico en el departamento del Huila, y la razón por la que se debe trabajar en pro de mejorar la condiciones prehospitalarias y hospitalarias, para brindar una mayor calidad de atención médicoquirúrgica a este tipo de pacientes, que permita optimizar al máximo el pronóstico posquirúrgico de los mismos, modificando variables como transfusión de hemoderivados, tiempo al inicio de la reparación quirúrgica, reparación multidis- 
ciplinaria y uso de ayudas imaginológicas diagnósticas, tratando así, por medio de una mejor implementación de técnica quirúrgica, disminuir una de las principales complicaciones posquirúrgicas: la amputación de una extremidad ${ }^{18-20}$. Es necesario recalcar la necesidad de continuar con estudios de tipo prospectivo relacionados con este tema, que permitan identificar factores y determinantes cruciales asociados a mejor pronóstico respecto al trauma arterial periférico en el Huila.

\section{Conclusión}

En este estudio se hallaron 54 casos de trauma arterial periférico, con una incidencia de I, $5 \%$ de las consultas por trauma al servicio de urgencias. Los principales mecanismos de trauma fueron las heridas por armas cortopunzante y cortocontundente; la región más afectada fueron las extremidades superiores e inferiores. Las intervenciones quirúrgicas fueron hechas en mayor medida por el servicio de Cirugía general, seguido de Cirugía vascular; el manejo principal fue la anastomosis término-terminal y el injerto de vena safena invertida. Las complicaciones más comunes fueron la trombosis arterial seguida de la amputación de extremidad, razón por la que se considera necesario desarrollar más estudios de tipo prospectivo, que permitan identificar claramente los factores asociados con el riesgo de amputación, de modo que se puedan definir estrategias dinámicas que permitan mejorar la morbimortalidad de estos pacientes en esta región del país.

\section{Referencias}

I. He JC, Clancy K, Schechtman D, Conrad-Schnetz KJ, Claridge JA. Traumatic vascular injuries: who are repairing them and what are the outcomes? Am J Surg. 20I6;2II:6I9-25. doi: I0.IOI6/j.amjsurg.2OI5.II.OII.

2. Liang NL, Alarcon LH, Jeyabalan G, Avgerinos ED, Makaroun MS, Chaer RA. Contemporary outcomes of civilian lower extremity arterial trauma. J Vasc Surg. 20I6;64:73I-6. doi: I0.IOI6/j.jvs.2016.04.052.

3. Morales-Uribe $\mathrm{CH}$, Sanabria-Quiroga AE, Sierra-Jones JM. Vascular trauma in Colombia: experience of a level I trauma center in Medellin. Surg Clin North Am. 2002;82:195-2Io. doi: IO.IOI6/Soo39-6IO9(03)ooI49-X.
4. Rojas S RA, Ramirez M, Camacho E, Alvarado R. Caracterización del trauma vascular periferico del hospital del Kennedy (tesis). Bogotá: Universidad Colegio Mayor de Nuestra Señora del Rosario. 2016.

5. Topal AE, Eren MN, Celik Y. Lower extremity arterial injuries over a six-year period: outcomes, risk factors, and management. Vascular Health and Risk Management. 20I0;6:IIO3-IO. doi: Io.2I47/VHRM.Si53I6.

6. Heldenberg E, Givon A, Simon D, Bass A, Almogy G, Peleg K. Civilian casualties of terror-related explosions: The impact of vascular trauma on treatment and prognosis. J Trauma Acute Care Surg. 2016;81:435-40. doi: I0.I097/TA.ooooooooooooII23.

7. DuBose JJ, Savage SA, Fabian TC, Menaker J, Scalea T, Holcomb JB, et al. The American Association for the Surgery of Trauma PROspective Observational Vascular Injury Treatment (PROOVIT) registry: multicenter data on modern vascular injury diagnosis, management, and outcomes. J Trauma Acute Care Surg. 2015;78:215-23. doi: I0.1097/TA.oooooooooooo0520.

8. Dunn JC, Kusnezov N, Schoenfeld AJ, Orr JD, Cook PJ, Belmont PJ, Jr. Vascular Injuries in Combat-Specific Soldiers during Operation Iraqi Freedom and Operation Enduring Freedom. Ann Vasc Surg. 2016;35:30-7. doi: I0.IoI6/j.avsg.20I6.0I.040.

9. Sisli E, Kavala AA, Mavi M, Sariosmanoglu ON, Oto O. Single centre experience of combat-related vascular injury in victims of Syrian conflict: retrospective evaluation of risk factors associated with amputation. Injury. 20I6;47:1945-50. DOI: IO.IOI6/j.injury.20I6.03.030.

Io. Espinoza R DP, Sotelo P. Trauma arterial de extremidades: resultados del manejo por el cirujano no especialista. Rev Chil Cir. 2002;54:225-30.

II. Sonneborn R, Andrade R, Bello F, Morales-Uribe CH, Razuk A, Soria A, et al. Vascular trauma in Latin America: a regional survey. Surg Clin North Am. 2002;82:18994. doi: Io.IoI6/Soo39-6IO9(03)ool48-8.

I2. Franz RW, Skytta CK, Shah KJ, Hartman JF, Wright ML. A five-year review of management. of upper-extremity arterial injuries at an urban level I trauma center. Ann Vasc Surg. 20I2;26:655-64. doi: I0.IOI6/j.jvs.2OII.0I.052.

I3. Kauvar DS, Sarfati MR, Kraiss LW. National trauma databank analysis of mortality and limb loss in isolated lower extremity vascular trauma. J Vasc Surg. 20II;53:I598-603. doi: IO.IOI6/j.jvs.20II.0I.056.

I4. Markov NP, DuBose JJ, Scott D, Propper BW, Clouse $\mathrm{WD}$, Thompson $\mathrm{B}$, et al. Anatomic distribution and mortality of arterial injury in the wars in Afghanistan and Iraq with comparison to a civilian benchmark. J Vasc Surg. 2012;56:728-36. doi: IO.IOI6/j.jvs.2012.02.048.

I5. Jiménez CE. Tratamiento endovascular del trauma vascular periférico. Rev Colomb Cir. 2012;27:290-7.

I6. Chong VE, Lee WS, Miraflor E, Victorino GP. Applying peripheral vascular injury guidelines to penetra- 
ting trauma. J Surg Res. 20I4;190:300-4. doi: Io.IOI6/j. jss.20I4.03.035..

I7. Schlickewei W, Kuner EH, Mullaji AB, Gotze B. Upper and lower limb fractures with concomitant arterial injury. J Bone Joint Surg Brit. 1992;74:18I-8.

I8. Fox N, Rajani RR, Bokhari F, Chiu WC, Kerwin A, Seamon MJ, et al. Evaluation and management of penetrating lower extremity arterial trauma: an Eastern Association for the Surgery of Trauma practice management guideline. J Trauma Acute Care Surg. 20I2;73(5 Suppl 4):S315-20. doi: I0.IO97/TA.oboI3e3I8270I8e4.
19. Kalish JA, Farber A, Homa K, Trinidad M, Beck A, Davies MG, et al. Factors associated with surgical site infection after lower extremity bypass in the Society for Vascular Surgery (SVS) Vascular Quality Initiative (VQI). J Vasc Surg. 20I4;60:I238-46. doi: I0.IOI6/j. jvs.20I4.05.012.

20. Perkins ZB, Yet B, Glasgow S, Cole E, Marsh W, Brohi $\mathrm{K}$, et al. Meta-analysis of prognostic factors for amputation following surgical repair of lower extremity vascular trauma. Br J Surg. 2015;IO2:436-50. doi: IO.IOO2/ bjs.9689. 